\title{
An Analysis on Measuring Graph patterns in Social Networks
}

\author{
Riaz Ahmed Shaikh ${ }^{1}$,Noor Ahmed Shaikh ${ }^{2}$, Muhammad Hanif Tunio ${ }^{3}$, Yumna Magsi ${ }^{4}$, Sajid Ahmed \\ Ghangro $^{5}$, Saima Shoro ${ }^{6}$ \\ Department Of Computer Science \\ ${ }^{1}$ Shah Abdul Latif University Khairpur Mir;s, Sindh, Pakistan, riaz.shaikh@ @alu.edu.pk \\ ${ }^{2}$ Shah Abdul Latif University Khairpur Mir;s, Sindh, Pakistan, noor.shaikh@ salu.edu.pk \\ ${ }^{3}$ Shah Abdul Latif University Khairpur Mir;s, Sindh, Pakistan, hanif.tunio@ salu.edu.pk \\ ${ }^{4}$ Shah Abdul Latif University Khairpur Mir;s, Sindh, Pakistan, yumna.magsi@ salu.edu.pk \\ ${ }^{5}$ Shah Abdul Latif University Khairpur Mir;s, Sindh, Pakistan, sajid.ghangro@ salu.edu.pk \\ ${ }^{6}$ Shah Abdul Latif University Khairpur Mir;s, Sindh, Pakistan, shorosaima @ gmail.com
}

\begin{abstract}
Concepts from graph theory might be useful in social networking for a variety of reasons. Graphs have recently been widely utilized in social networks for a variety of reasons, including modelling and analysis of SN architecture, SN operation modelling, SN user analysis, and a variety of other topics.A graph consists of nodes; just like that, a social media is a kind of social network where a node is defined by each person or organization. Via common interests, relationships, mutual friends, expertise, common dislikes, views etc., these nodes in a social network are interdependent on each other. The application and implications of graph theory on social networking, social network analysis, Interpersonal relations, and social network properties are focused in this paper. The main goal of his study was to relate the social networks with graph theory.An Analysis of a few Facebook users, needed for social media structural analysis, is also included.
\end{abstract}

Key words: Graph Theory, Social Network, Facebook, Twitter.

\section{GRAPH}

Graph is a pictorial representation of statistical data of a functional representation of the variable. [1] It shows the general tendencies in quantitative behavior of data therefore serve as a predictive function. A graph is used to represent the network of edges and vertices which are interconnected with each other. [2][3][4] Shown in figure no: 1

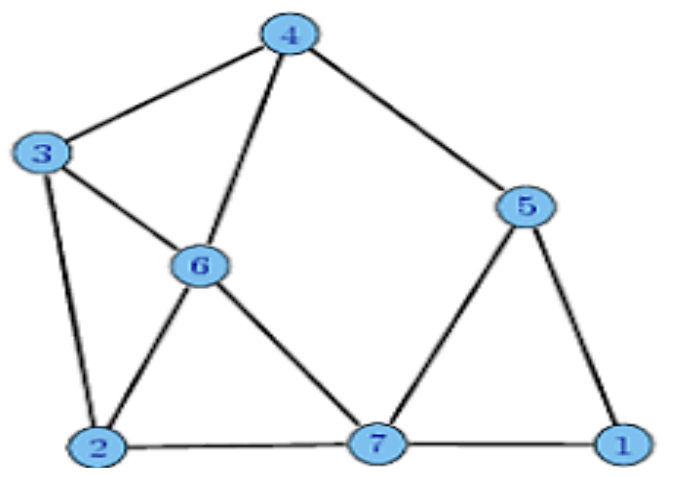

The vertices are basically the nodes of the networks and edges are the connection between those nodes. The edges are of two types

1. Directed Graph

2. Undirected Graph (Shown in Figure 2)

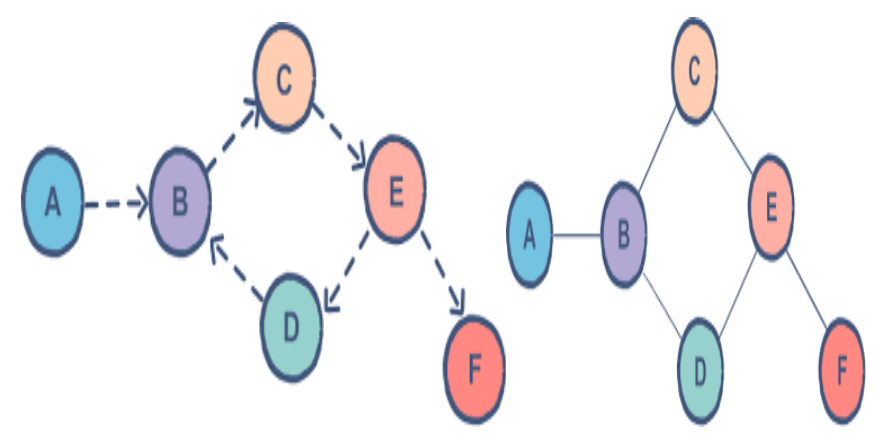

Figure 2 Directed and Undirected Graph

Directed grapn snows the direction or patn trom one node to another however undirected graph does not 
describe the particular path towards the nodes. On the basis of presence and absence of cycles the graphs can be classified as:

\section{Cyclic}

2. Acyclic [5] (shown in figure 3)
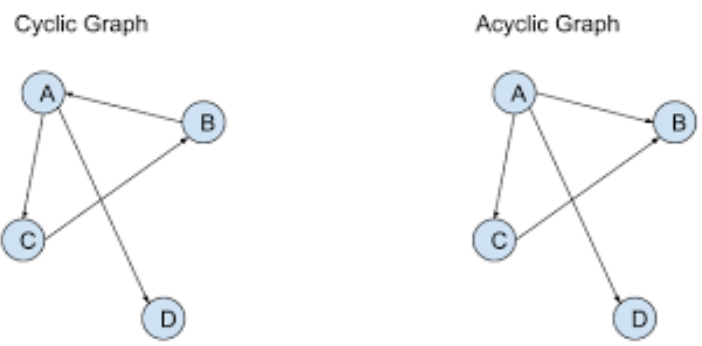

There Figure 3 Cyclic and Acyclic Graph ry but in social networking it is gaining popularity day by day.

This paper reveals the graph with reference to social networking.

\section{NETWORKS}

Depending on the branch of mathematics used, there are many ways of formally describing a network.

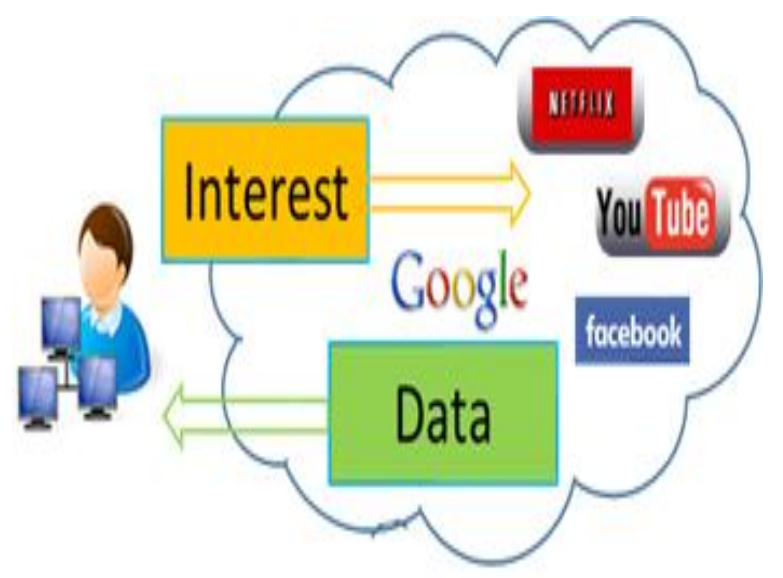

Figure 4 Networks

A social network is conceptualized as a graph, that is, a collection of vertices (or nodes, units, points) representing social entities or objects and a set of lines representing one or more social connections between them. The most common and versatile meaning is derived from the theory of graphs. [5]
On the basis of nodes contents network can be divided into two types: (Illustrated in Figure 4)

\subsection{Social and Economic Networks}

It includes the group of people interconnected with each other's through some patterns of communications.

Such as Facebook, Twitter, WhatsApp, Instagram and business Networks etc.

\subsection{Information Networks}

Information Networks describes the connections between information objects. Such as Semantics, Word Wide Web.

Whereas the main focus of this paper is the social networks particularly Facebook and Twitter.

\subsection{Social Networks}

A social network is a website that brings people together to chat, connect or make new friends with ideas and interests. Social networking is referred to as this form of communication and sharing.[6]

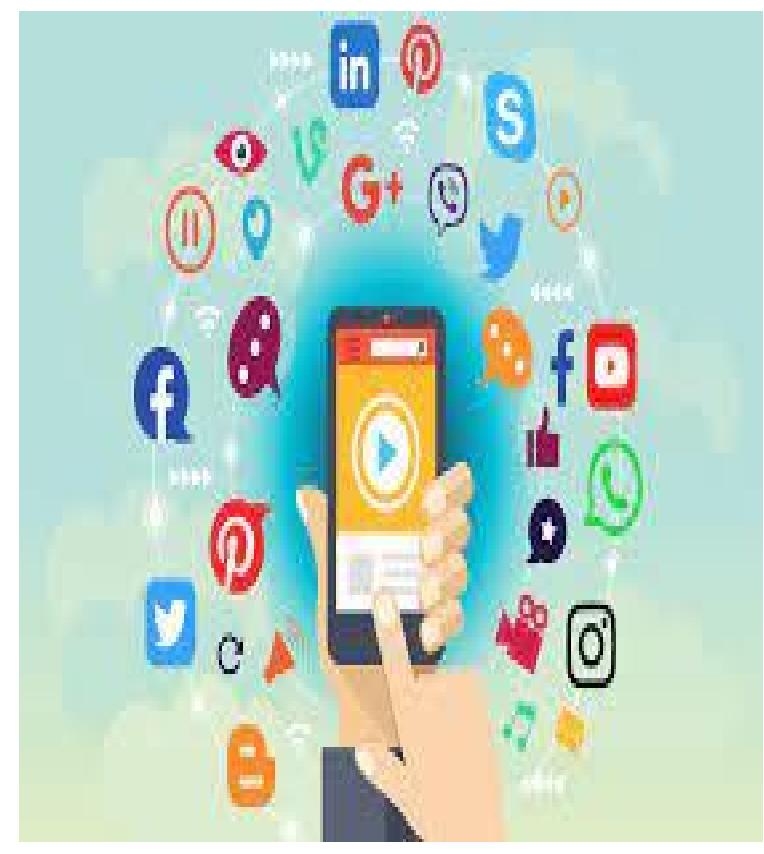

Figure 5 Social Networks

Social networking sites contain content produced by hundreds or even millions of different people, unlike conventional media created by no more than ten individuals. Figure 6 shows the network of friends. 


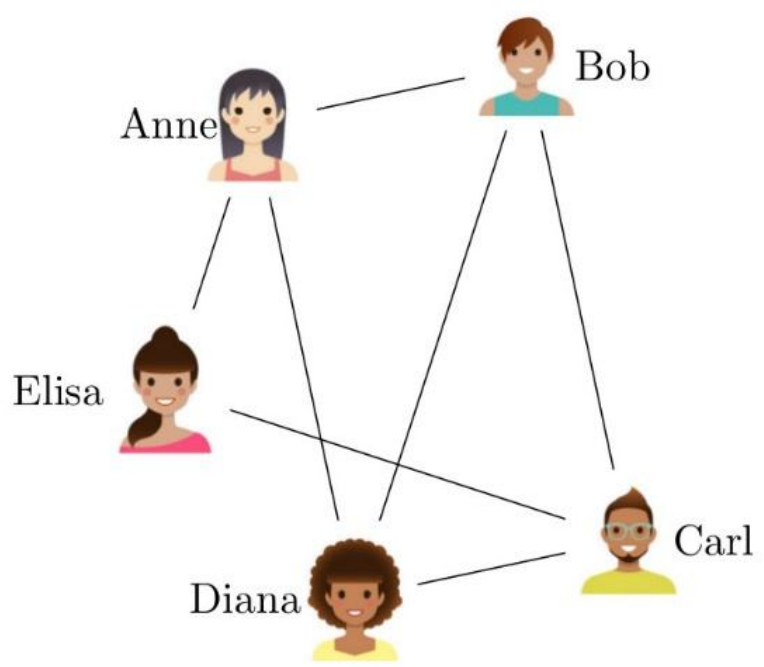

Figure 6 Network of Friends

\section{GRAPH THEORY AND SOCIAL NETWORKS}

The graph theory covers the large area of Social Networks. Where the users are considered as vertices or nodes whereas the relations such as common likes and mutual friends are considered as edges. [7][8][8][9][10][11] Figure 7 shows the graph of social networks.

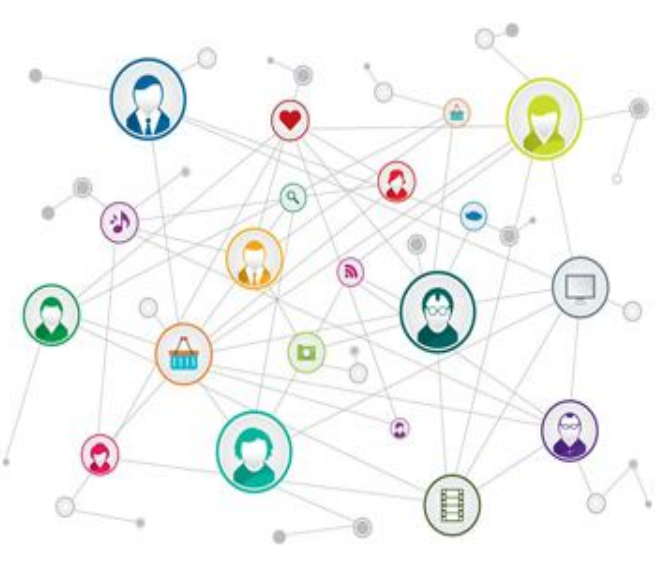

Figure 7 Graph of Social Networks

\subsection{Graph Theory in Facebook:}

As Facebook is the major source of communication now a days. The Facebook provides an interesting way to connect with your friends, family and other strangers by liking their posts, tagging them on timeline and by commenting on their posts. With reference to graph theory the users are vertices of graph whereas the tags, comments and likes are known as edges of the graph.[6] Figure 8 shows the graph of Facebook.

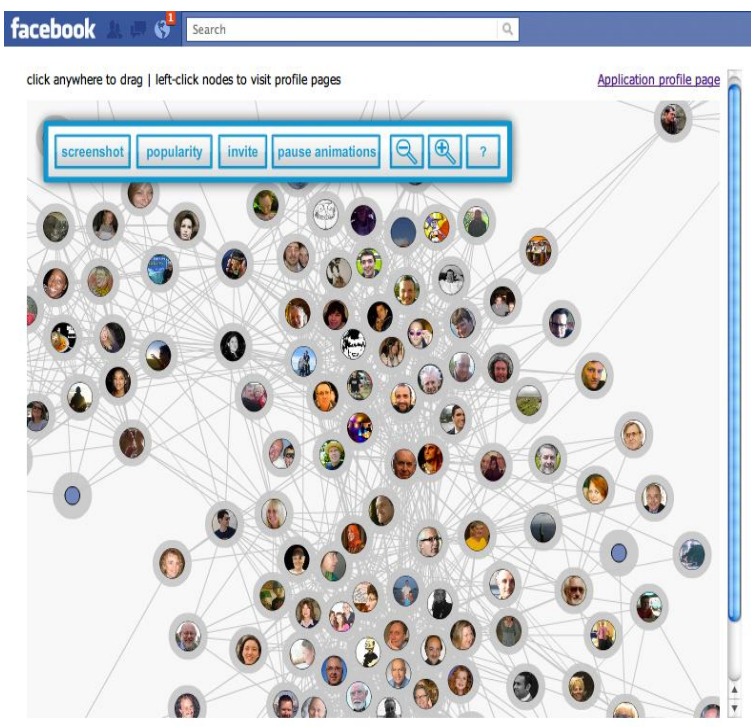

Figure 8 Graph of Facebook

\subsection{Graph Theory in Twitter:}

The persons or users are considered as nodes and their following is known as edges. Shown in figure 9.

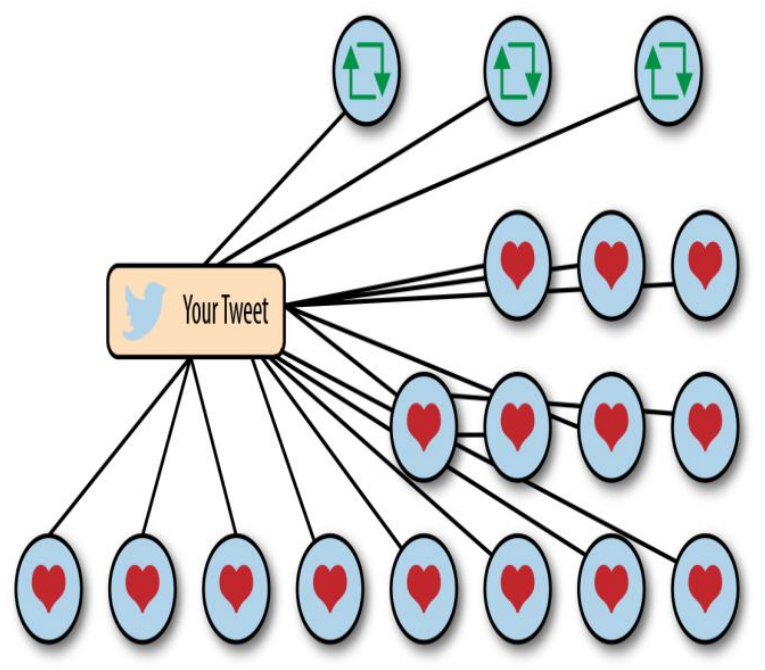

Figure 9 Graph of twitter

\section{MEASURING NETWORKS ON FACEBOOK}

The size of Facebook networks is reflected by this case study. For the course of a month, few random 
users have been studied, and the relationship is categorized into four distinct network patterns [7]

\subsection{All Friends}

This network is the list of all a user's friends, so this is the largest of all representations.

\subsection{Reciprocal communication}

This representation indicates the reciprocal communication between the two parties, where there is a mutual exchange of information between the two parties, creating this sort of network. [8]

\subsection{One-way communication}

It is made up of individuals with whom a user has interacted.

\subsection{Maintained Relationships}

This relationship trend consists of individuals whose profile to maintain commitment has been reviewed by the consumer more than once. [9] [10] The blue line shows the number of mutual relationships in the Figure: 3, the blue line shows the one-way relationships, and the blue line shows that yournetwork size is a function of passive relationships.
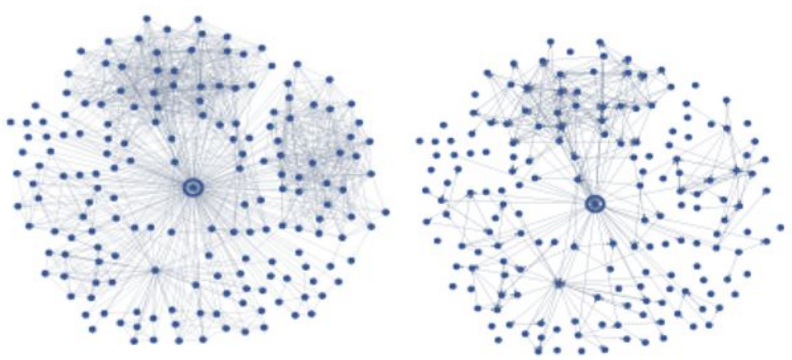

All Friends

Maintained Relationship
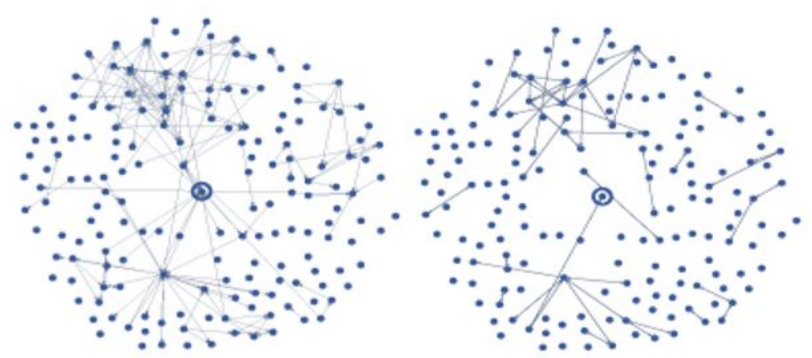

Oneway Communication

Mutual Communication

Figure 10 Network patterns of Facebook Users

\section{GRAPH PARTITIONING}

Graphs are frequently utilized to represent an application software by numerous scientists or researchers. The challenge of partitioning a graph is entirely algorithmic. It aids in the reduction of the complexity of large graphs as well as the introduction of parallelization.Graph partitioning is needed in a variety of applications, including social networks, road networks, air traffic management, picture analysis, and so on. Scientific computing, dividing various phases of VLSI design circuits, job scheduling in multiprocessor systems, and other uses of graph portioning issues are only a few examples. The goal of graph partitioning is to separate nodes into many disjoint sections in order to minimize the stated objective function. Although the issue of optimum network partitioning is NP-complete, several approximation methods have been developed to address it.

There are two types of graph partitioning:

\subsection{Constraints Partitioning}

This partitioning has portions that are all the same size.

\subsection{Unconstrained Partitioning}

This type of partitioning has sections of varying sizes. For graph partitioning, a number of methods have been developed. Geometric partitioning, Spectral partitioning, and Multi-level graph partitioning are three of the most important algorithms. [14] Graph partitioning is shown in figure 11.
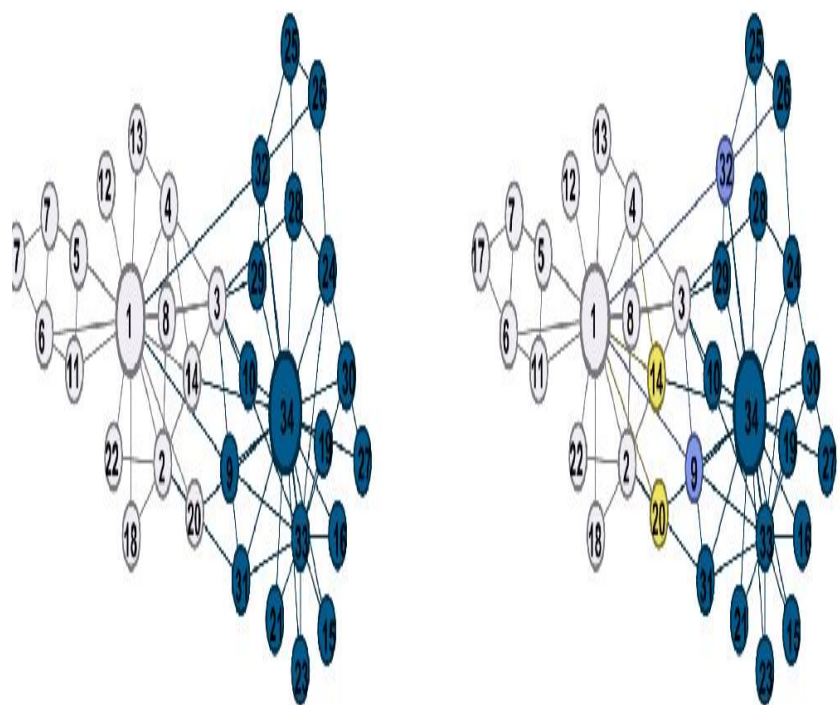

Figure 11 Constraint and Unconstraint partitioning

The graph is bisected in Geometric partitioning by using the coordinates that are acquired if nodes of a graph are available in space. 
The vertices that are geographically close to each other are grouped together in this division. The associability of the graph is determined by determining the Eigen vectors with regard to the second lowest Eigen value of the Laplacian matrix L corresponding to the graph in Spectral partitioning. This bisection approach is extremely time consuming, yet it is not practical for big graphs.

Multi-level partitioning outperforms traditional graph partitioning techniques. This technique effectively solves constrained graph partitioning issues. The basic concept is to divide huge networks into $\mathrm{k}$ sections, group the vertices into a group, and deal with this group of vertices instead of individual vertices.

It is divided into three stages:

\section{Coarsening,}

2. Initial partitioning,

3. Partition refining.[14][15] (discussed in figure 12)

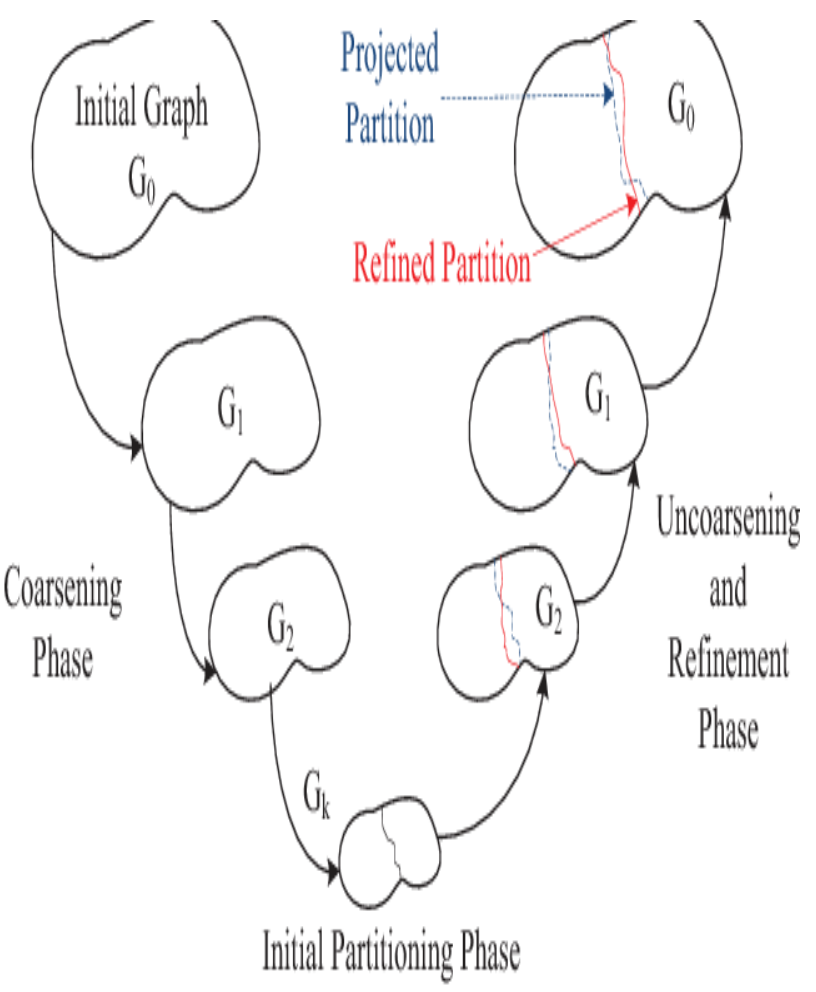

Figure 12 Multilevel Partitioning

A graph is a mathematical structure that depicts the relationships between different items. The vertices, nodes, or points of a graph are connected by lines, arcs, or edges. It is possible for a graph to be directed or undirected. $\mathrm{G}=(\mathrm{V}, \mathrm{E})$, where $\mathrm{V}$ is a collection of vertices and $\mathrm{E}$ is a set of edges, is a graph.

A graph is represented using adjacency lists, adjacency matrices, incidence matrices, and other data structures.Individuals or organizations are represented as nodes in a social network, while relationships between individuals or organizations are represented by edges.A collection of tools for studying the structure of whole social entities is provided by social networks.Different degrees of analysis exist, and they aren't always mutually incompatible.

Generally there are three levels:

\subsection{Micro level}

It usually starts with a person or a small group of individuals at this level.

\subsection{Meso level}

This level is halfway between micro and macro. It demonstrates the link between the micro and macro scales. Meso-level networks are networks with a low density.

\subsection{Macro-level networks}

These are large-scale networks that track relationships across enormous populations.

These are more complicated networks illustrated in Figure 13.

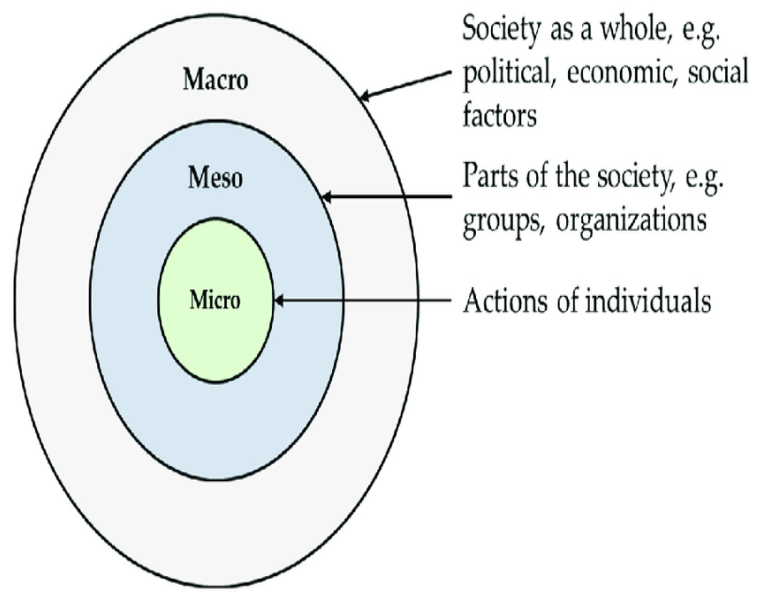

Figure 13 Levels of graph

\section{CONCLUSION}

This paper addressed the different aspects of graphs and their basic properties. Graphs are used in social networks that are growing in sophistication with the progress of social media. Different algorithms are still being developed by researchers to better 
understand social networks and to partition graphs to reduce their complexity and to better understand networks. We have given a survey of the work done in the field of social networks that uses graph theory ideas in this paper. By evaluating graph characteristics and selecting the correct mix of graphs based on their challenge, the contribution can help many academics from diverse viewpoints.Beginner researchers can benefit from the well-summarized possible applications of graph theory, which can help them understand its ideas and current use in a variety of SN areas. The application and implications of graph theory on social networking, social network analysis, Interpersonal relations, and social network properties are focused in this paper. The main goal of his study was to relate the social networks with graph theory.An Analysis of a few Facebook users, needed for social media structural analysis, is also included.

\section{REFERENCES}

1. Marcin Mince, EwaNiewiadomska-Szynkiewicz, "Application of Social Network Analysis to the Investigation of Interpersonal Connections", February 2012.

2. Kimball Martin, "Graph Theory and Social Networks", April 30, 2014.

3. D. Easley, J. Kleinberg. Networks, Crowds, and Markets: Reasoning About a Highly Connected World. Cambridge University Press, 2010.

4. T.h. Cormen, C.E. Leiserson, R.L. Rivest, C. Stein, Introduction to Algorithms (2nd ed.), MIT Press and McGraw-Hill, ISBN 0-262-53196-8, 2001.

5. AlexandruCostan, "Graph Theory and Social Networks",

6. https://www.uva.nl/binaries/content/documents/p ersonalpages/ n/o/w.denooy/en/tab-one/tabone/cpitem\%5B26\%5D/asset?1355372751494.

7. Daron Acemoglu and AsuOzdaglar, "Networks, Lecture 2: Graph Theory and Social Networks", September 14, 2009.

8. Jayson Rome, "Graph Triangulation", October 14, 2002.

9. S. Wasserman, K. Faust, Social Network Analysis in the Social and Behavioral Sciences, Social Network Analysis: Methods and Applications, Cambridge University Press, 1994, pp. 1-27.

10. J. Farganis, Readings in Social Theory: the Classic Tradition to Post-Modernism, McGrawHill, New York, 1993.

11. G. Simmel, Die Grosstädte und das Geistesleben (The Metropolis and Mental Life), Dresden: Petermann, 1903.
12. J. Levy Moreno, Who shall survive?: Foundations of sociometry, group psychotherapy and sociodrama, Beacon House, 1953.

13. M. McPherson, L. Smith-Lovin, J.M. Cook, Birds of a feather: homophily in social networks, Annual Review of Sociology, Vol. 27, 2001, pp. 415-444.

14. G. Robins, P. Pattison, J. Woolcock, Small and Other Worlds: Global Network Structures from Local Processes. American Journal of Sociology (AJS), Volume 110, Number 4 (2005), 894-936.

15. Kaur, Chhinder, and Anand Sharma. "Sentiment Analysis of Tweets on Social Issues using Machine Learning Approach." International Journal 9, no. 4 (2020). 